\title{
INDEX OF VOLUME 58
}

\section{Subjects:}

\section{INDEX OF ABSTRACTS}

Algebra and Theory of Numbers, 39, 51, 62, 152, 164, 380, 448, 472, 493, 559, 619.

Analysis, 41, 56, 66, 153, 173, 385, 453, 476, 494, 566, 630.

Applied Mathematics, 46, 58, 68, 154, 190, 389, 462, 486, 499, 572, 651.

Geometry, 59, 69, 155, 196, 390, 464, 488, 501, 573, 655.

Logic and Foundations, 198, 391, 464, 490, 501, 659.

Statistics and Probability, 60, 199, 391, 465, 502, 660.

Topology, 48, 60, 69, 155, 201, 393, 465, 491, 502, 573, 661.

Authors:

Agmon, S., 173; Ainsworth, O. R., 58; Albert, A. A., 448; Allen, A. C., 453; Allen, E. F., 488; Ambrose, W., 453; Anderson, D. R., 559; Anderson, R. D., 174, 201, 393, 465, 661; Andrushkiw, J., 174, 385, 448; Ankeny, N. C., 164, 560; Antosiewicz, H. A., 630; Apostol, T. M., 62, 559; Arms, R. J., 174; Aronszajn, N., 175, 630; Arsove, M. G., 566; Aucoin, A. A., 39, 41.

Bagemihl, F., 619; Ball, B. J., 201; Barrett, J. H., 453; Bartholomay, A. F., 201; Bartle, R. G., 175; Baum, W. R., 202; Beckenbach, E. F., 453; Becker, H. W., 39, 39, $63,165,391,392,466,466,560,560,561,561,620,620,651,661,696$; Bellman, R. E., 494; Bennett, D. W., 501; Bergman, S., 454; Bergmann, H. G., 462; Berkovitz, L. D., 66; Berman, G., 489; Bernardi, S. D., 385; Bernhart, A., 155, 661; Bernstein, B. A., 565; Bers, L., 175; Beth, E. W., 502; Blackett, D. W., 620; Blackman, J., 454, Blair, R. L., 472, 621; Blakers, A. L., 202, 466; Blanch, G., 46, 572; Blanchfield, R. C., 69; Block, H. D., 476, 486; Block, I. E., 631; Blumenthal, L. M., 380, 501; Boas, R. P., 175, 386, 454, 476, 566, 631; Bohun-Chudyniv, V., 165, 380, 621; Bonsall, F. F., 175; Boone, W. W., 40; Bott, R., 202, 203; Bourgin, D. G., 489, 491; Bourne, S., 165; Bram, J., 631; Brauer, A. T., 40, 51, 622; Brauer, G., 631; Brenner, J. L., 622; Browder, F. E., 176, 176, 176, 177, 177, 203, 386, 386, 454, 455, 632, 632, 632; Brown, B., 203; Brownell, F. H., 494; Brunk, H. D., 177, 487; Buck, R. C., 467, 467, 566, 632; Burgess, C. E., 661; Burrill, C. W., 632; Butzer, P. L., 633.

Cahn, A. S., 487; Calabi, E., 655; Calder6n, A. P., 477, 477; Cameron, R. H., 177, 477; Campbell, H. E., 622; Cargal, B., 476; Carlitz, L., 51, 51, 51, 166, 166, 166, 380, 381, $381,381,381,448,448,449,449,449,561,562,562,562,562,622,623,623,623,623$; Carruth, P. W., 449; Carter, W. C., 46; Cesari, L., 178; Chen, K. T., 166, 472; Chen, Y. W., 153, 455; Chover, J., 633; Chowla, S., 167; Chrestenson, H. E., 567; Chung, K. L., 199, 660; Churchill, R. V., 634; Civin, P., 66, 567; Clark, R. A., 190; Clarke, F. M., 381, 473; Coddington, E. A., 42, 42, 47, 455, 456; Cohen, A. C., 60; Cohen, E., 167, 450, 450, 450; Cohen, H., 60; Cohn, H., 167, 623; Cohn, R. M., 456; Comfort, E. G. H., 634; Conkling, R. M., 386, 656; Cooke, K. L., 567; Cooperman, P., 456; Copeland, A. H., 167, 473; Court, N. A., 155; Cowling, V. F., 477; Coxeter, H. S. M., 624; Crispin, J. W., 463; Currie, J. C., 52; Curtis, C. W., 562; Curtis, M. L., 204, 467.

Danskin, J. M., 178; Darling, D. A., 42; Davis, A. C., 63, 382, 450; Davis, A. S., 52; Davis, C., 382; Davis, R. B., 387, 457; Davis, R. L., 659; DeCicco, J., 193, 634, 641, 652, 657; Dekker, D. B., 69; Devinatz, A., 478; DeVogelaere, R. J., 191; Diaz, J. B., 191; Dietrich, V. E., 52; Diliberto, S. P., 467; Dilworth, R. P., 70; Dolph, C. L., 634; Donoghue, W. F., 478; Douglas, Jesse, 40, 40; Douglas, Jim, 200; Douglis, A., 457; Drandell, M., 69; Dresher, M., 178; Dressel, F. G., 57; Dubois, D. W., 152, 624; Duffin, R. J., 634, 652; Duncan, D. G., 63; DuVal, P., 59; Dye, H. A., 563, 567.

Eaves, J. C., 52; Eckmann, B., 382; Edrei, A., 174; Elliott, H. M., 43; Elliott, J., 179; Ellis, D. O., 53, 53, 196, 386, 393, 573, 656, 662; Ellis, J. W., 56; Erdös, P., 619; Estill, M. E., 467, 662; Evans, R. L., 478; Evans, T., 167, 382; Ewing, G. M., 156, 487. 
Fadell, E. R., 491; Faulkner, F. D., 652; Feit, W., 473; Feller, W., 179, 179; Fettis, H. E., 487; Fialkow, A. D., 191, 192; Ficken, F. A., 192; Finkbeiner, D. T., 180; Finn, R. S., 635; Finzi, A , 196; Flanders, H., 63; Fleming, W. H., 635; Forsythe, G. E., 502; Fort, M. K., 662; Fort, T., 57; Frame, J. S., 474; Friedman, B., 652; Friedrichs, K. O., 635; Fulks, W. B., 636; Fuller, L. E., 474; Fullerton, R. E., 57, 180; Fulton, C. M., 573.

Gaddum, J. W., 197; Gál, I. S., 180, 636; Gale, D., 656; Gergen, J. J., 57; Germond, H. H., 180; Gerst, I., 191, 192; Ghaffari, A., 43, 47, 66, 68, 154, 155, 389, 457, 568; Gillman, L., 178; Ginsburg, S., 479, 479, 479, 479, 636, 637, 637, 637; Givens, W., 53; Goffman, C., 637, 638; Goheen, H. E., 192; Goldberg, S., 181, 458; Goldberg, S. I., 663, 663; Goldhaber, J. K., 451; Goldman, O., 624; Good, R. A., 624; Goodman, A. W., 638; Gormsen, S. T., 656; Gottschalk, W. H., 383; Graves, R. L., 479; Green, J. W., 181, 387, 458; Greer, E. V., 153; Griffin, E. L., 480, 480; Grove, V. G., 489, 489.

Hadnot, B. F., 57; Haimo, F., 168, 625; Hall, M., 41, 451; Halmos, P. R., 638; Hammer, P. C., 197, 197, 198, 198, 480, 481, 490, 491, 573, 573; Hammersley, J. M., 639; Hamstrom, M. E., 204, 663, 663; Harary, F., 167, 168, 473, 664; Hausner, M., 563; Hayes, C. A., 181; Hayes, R. M., 653; Haynsworth, E. V., 53; Helson, H., 387; Henriksen, M., 168, 625; Herstein, I. N., 383, 383, 474, 625; Herzog, F., 639, 639; Hewitt, E., 639; Higman, D. G., 626; Hildebrand, F. B., 193; Hirsch, W. M., 200; Hirschman, I. I., 481; Hodge, P. G., 68, 499; Hodges, J. L., 660; Hohn, F. E., 653; Hopkins, H. G., 193; Horn, A., 64; Hostetter, I. M., 568; Hsiung, C. C., 656; Hu, S. T., 60, 70, 204, 393, 393, 574, 664; Huff, G. B., 54; Huff, W. N., 153; Hughes, D. R., 624; Hull, R., 383; Hutcherson, W. R., 656, 657.

Ikenberry, E., 59, 481; Isbell, J. R., 625.

Jackson, J. R., 70, 626; James, R. D., 640; John, F., 640; Johnson, R. E., 475, 626; Johnson, W. E., 640; Jones, B. W., 169; Juncosa, M. L., 464.

Kadison, R. V., 43, 181; Kainen, A. J., 649; Kakoris, L. A., 169; Kalicki, J., 64, 626; Kamel, H., 391; Kampé de Fériet, J., 67; Kaplansky, I., 169, 627; Kasner, E., 193, 390, 641, 657; Katz, L., 627; Katz, S., 653; Kaufman, H., 463; Kelly, L. M., 657; Kelly, P. J., 501; Kiefer, J. C., 465; Kimball, W. S., 641; Klamkin, M. S., 44, 67, 182, 568; Klee, V. L., 174, 204, 205, 390, 568; Kleene, S. C., 198; Klein, G., 458; Kleinfeld, E., 475; Kleinman, R. E., 463; Klimczak, W. J., 641; Koehler, F., 487; Kotik, J., 462; Kozin, F., 627; Krzywoblocki, M. Z., 488, 488; Kundert, E. G., 642, 642, 665; Kyle, R. H., 467, 468, 468.

Lanczos, C., 500; Lang, S., 384; Lapidus, L., 657; Lax, P. D., 182 ; Lee, E. H., 193; Lee, J. R., 482; Lehmer, D. H., 563; Leighton, W., 182, 459; Leipnik, R. B., 482, 569; Lepson, B., 183; Leslie, R. T., 44; LeVeque, W. J., 627, 627, 628, 628; Levinson, N., 42, 569; Levit, R. J., 54; Levitzki, J., 628; Lewis, D. J., 384, 475; Liao, S. D., 492; Lin, C. C., 194; Lindgren, B. W., 177; Lipschutz, M. A., 392, 660; Lister, W. G., 169; Litoff, O. I., 564, 564; Livesay, G. R., 492; Livingston, A. E., 569, 642, 642; Long, W. C., 649; Lorch, E. R., 570; Lorch, L., 183; Lorentz, G. G., 183; Love, E. R., 44; Lukacs, E., 200, 465; Luke, Y. L., 67; Lumer, G., 638.

McCulley, W. S., 649; McKinsey, J. C. C., 659; McLaughlin, J. E., 475, 628; MacNearney, J. S., 44; Macon, N., 54; Maharam, D., 642; Marden, M., 175; Markus, L. F., 459; Marlow, W. H., 48, 49; Martin, M. H., 191; Martin, W. T., 177; Massera, J. L., 44; Maxfield, J. E., 564, 564; Maxfield, M. W., 564; Medlin, G. W., 55; Miles, E. P., 57; Milgram, A. N., 630; Miller, K. S., 48, 462; Mills, W. H., 170; Minagawa, T., 464, 658; Mirkil, H., 643; Mitchell, Benjamin Ernest, 59, 658; Mitchell, Benjamin Evans, 55, 644; Mitchell, J. M., 482; Mittleman, D., 390, 658; Modigliani, F., 653; Moise, E. E., 205, 205, 206, 206; Montgomery, D., 665; Moore, C. N., 45; Moore, J. C., 468, 469, 469, 469, 665, 666; Mordell, L. J., 643; Morelock, J. C., 657; Morgenthaler, G. W., 482, 483; Morrison, D. R., 55, 493; Mostert, P. S., 666; Mostow, G. D., 
206; Motzkin, T. S., 41, 153, 183, 184, 494, 653, 658; Moulton, E. J., 184; Moy, S. T. C., 660; Murray, F. J., 462; Myers, W. M., 483.

Nehari, Z., 643; Newton, T. A., 58; Nikodým, O. M., 207, 570; Nomizu, K., 492; Norris, M. J., 565; Novak, I. L., 464.

O’Brien, G. G., 194; Olkin, I., 627; O'Neill, B., 502; Osborn, R., 649.

Paige, L. J., 501; Pan, T. K., 390, 501, 659; Parker, W. L., 565; Parker, W. V., 644; Payne, L. E., 47, 194, 654; Payne, M. H., 654; Pearson, E. H., 55; Peiser, A. M., 644, 653; Pell, W. H., 195; Penez, J. L., 184; Pennisi, L. L., 644, 644; Pepper, P. M., 170; Peters, G. O., 185; Pettis, B. J., 58; Phillips, R. S., 495, 495; Pierce, R. S., 71; Pipes, C. J., 154; Piranian, G., 639, 639; Pitcher, E., 666; Porcelli, P., 645; Prager, W., 68; Protter, M. H., 185, 459, 645; Pursell, L. E., 666, 667.

Rabson, G., 645; Rad6, T., 464, 464, 574, 658; Rainville, E. D., 153, 185; Redheffer, R. M., 494, 496, 496, 496, 498, 500, 500; Reichelderfer, P. V., 574; Reid, W. P., 496; Reissner, E., 190, 195; Reiter, H. J., 170, 171, 171, 646; Resch, D., 488; Reschovsky, H., 390; Reynolds, C. N., 207; Rice, H. G., 199, 199; Richardson, M., 470; Rickart, C. E., 451; Roberts, J. H., 459; Robinson, G. de B., 171; Rosen, D., 460; Rosenberg, A., 475; Rosenblatt, M., 660; Rosenbloom, P. C., 185, 483, 484, 646; Rosenfeld, L., 189; Rosenlicht, M. A., 384, 451, 655; Royden, H. L., 185, 646; Royster, W. C., 484; Rudin, W., 186, 646; Rutledge, W. A., 481.

Saltzer, C., 186, 654; Samuelson, P. A., 654; Sanders, J., 186, 195, 647; Sard, A., 187 ; Sario, L., 45, 187, 496, 497, 497, 498, 498; Saxon, D. S., 487; Schafer, R. D., 452; Schäffer, J. J., 638; Schatz, J. A., 647; Schenkman, E., 171, 629; Scherk, P., 64, 69; Schoenberg, I. J., 183, 184, 653, 658; Schuepbach, D., 172; Schurrer, A. L., 647; Schweitzer, A. R., 384, 391, 391, 490, 490, 490; Schwerdtfeger, H. W. E., 476; Scott, D. S., 626; Seidenberg, A., 494; Selfridge, R. G., 572 ; Selmer, E. S., 64; Shaffer, D. H., 652; Shanks, D., 452; Shanks, M. E., 667; Shapiro, H. S., 570; Shapiro, V. L., 484, 485; Sharp, H., 667; Sheffer, I. M., 460; Sherman, S., 187, 187, 188, 196, 460; Shniad, H., 648; Siegel, K. M., 463; Silverman, E., 648; Sinclair, A., 460; Singer, I. M., 453; Slater, M. L., 188; Smiley, M. F., 485; Sobczyk, A., 197, 198, 198, 480, 481, 490, 491, 571, 573; Sorgenfrey, R. H., 503; Spencer, D. E., 196, 655; Spencer, G. L., 46; Sprinkle, H. D., 573; Stein, M. L., 388, 655; Stein, S. K. B., 207; Steinberg, R., 498; Stelson, H. E., 629; Sternberg, R. L., 188, 389, 463; Stone, A. H., 667, 667; Stone, W. M., 572; Straus, E. G., 65; Strebe, D. D., 667; Strother, W. L., 60; Sumner, D. B., 461; Suppes, P., 659; Swingle, P. M., 470; Szász, O., 189, 465.

Taam, C. T., 485, 485, 648; Tamari, D., 565, 565; Tarski, A., 65, 65, 172; Taussky, O., 41; Thompson, F. B., 65; Thron, W. J., 649; Tinnappel, H., 668; Titt, E. W., 649; Titus, C. J., 649, 649; Tomber, M. L., 452; Toralballa, L. V., 45; Tornheim, L., 650; Tukey, J. W., 502. 156.

Uhlenbeck, G. E., 168; Uhrich, G. E., 571; Ullman, J. L., 499, 571, 650; Utz, W. R.,

Vaught, R. L., 66; Volkmann, B., 385, 452.

Wagner, D. H., 41; Wahab, J. H., 56; Walker, G. L., 629; Wall, H. S., 650; Wallace, A. D., 61, 629, 668, 668, 668; Walsh, J. L., 189, 189, 388, 388; Wang, H. C., 207; Warga, J., 388; Warschawski, S. E., 484; Wasow, W. R., 153, 499; Waterman, D. R., 486, 486; Wechsler, M. T., 207; Weil, H., 201; Weinberger, H. F., 48, 190, 463; Weinstein, A., 194, 650; Wendel, J. G., 563; Wermer, J., 651; Whitehead, G. W., 208; Whyburn, G. T., 669; Wiegmann, N. A., 172; Wilansky, A., 190, 461, 572; Wilcox, L. R., 630; Willoughby, R. A., 56; Wolf, F., 67, 68, 461; Wolfowitz, J., 465, 465; Worthington, L. G., 649.

Yang, C. T., 61, 470; Yood, B., 389; Young, D. M., 189, 388, 464; Young, G. S., 649; Young, J. W., 58; Young, L. C., 635, 651.

Zadeh, L. A., 48; Zarantonello, E. H., 46; Zelinsky, D., 173; Zemmer, J. L., 173; Zippin, L., 665. 
INDEX OF REPORTS OF MEETINGS AND MISCELLANEOUS ARTICLES

Begle, E. G. Memorandum to members, 1.

Begle, E. G. Publication charges, 115.

Begle, E. G. Reports of meetings of the American Mathematical Society: Summer Meeting in East Lansing, 612.

Begle, E. G. See Heins, A. E.

Bliss, G. A. See Graves, L. M.

Bochner, S. Harald Bohr, April 22, 1887-January 22, 1951, 72.

Bohr, H. See Bochner, S.

Brahana, H. R. Errata to George Abram Miller, 1863-1951, 696.

Cartan, E. See Chern, S. S.

Chern, S. S., and Chevalley, C. Elie Cartan and his mathematical work, 217.

Chevalley, C. See Chern, S. S.

Cohen, L. W. Reports of meetings of the American Mathematical Society: October Meeting in Washington, 38; Annual Meeting of the Society, 157; February Meeting in New York, 379; A pril Meeting in New York, 439.

Graves, L. M. Gilbert Ames Bliss, 1876-1951, 251.

Heins, A. E., and Begle, E. G. Fifth Symposium on Applied Mathematics, 557.

Puckett, W. T. Reports of meetings of the American Mathematical Society: December Meeting in Pasadena, 62; May Meeting in Fresno, 493; June Meeting in Eugene, 559.

Whyburn, W. M. Reports of meetings of the American Mathematical Society: November Meeting in Auburn, 50.

Youngs, J. W. T. Reports of meetings of the American Mathematical Society: November Meeting in Norman, 152; April Meeting in Chicago, 471.

\section{INDEX OF BOOK REVIEWS}

Activity analysis of production and allocation. Ed. by T. C. Koopmans. J. Wolfowitz, 395.

Advances in applied mechanics. Vol. 2. Ed. by R. von Mises and T. von Kármán. C. Truesdell, 403.

Atti del terzo Congresso dell Unione Matematica Italiana tenuto in Pisa nei giorni 23-26 Settembre 1948, 103.

Banach, S. Mechanics. Trans. by E. J. Scott. E. J. Moulton, 399.

Barcan Marcus, R. See Curry, H. B.

Behnke, H. See Bergman, S.

Bell, E. T. See Laplace, P. S.

Bergman, S. The kernel function and conformal mapping. H. Behnke, 76.

Bieberbach, L. Einfilhrung in die Funktiontheorie. M. Heins, 519.

Birkhoff, G. Hydrodynamics, a study in logic, fact, and similitude. Errata. J. J. Stoker, 696.

Blaschke, W. See Garnier, R.

Boas, R. P. See Cantor, G., Herland, L., Laplace, P. S.

Bochner, S. See Halperin, I., Schwartz, L.

Bouwkamp, C. J. See Tables relating to Mathieu functions.

de Broglie, L. Problemes de propagations guidées des ondes électromagnétiques. A. E. Heins, 100.

Buck, R. C. See Cooke, R. G.

Busemann, H. See Finsler, $P$.

Cantor, G. Contributions to the founding of the theory of transfinite numbers. Trans. by P. E. B. Jourdain. R. P. Boas, 682.

Casorati, F. Opere, 103.

Churchill, R. V. See Tranter, C. J. 
Coxeter, H. S. M. See Hodge, W. V. D.

Colloque de Topologie. (Espaces fibrés). W. S. Massey, 278.

Cooke, R. G. Infinite matrices and sequence spaces. R. C. Buck, 272.

Courant, R. Dirichlet's principle, conformal mapping, and minimal surfaces. With an appendix by M. Schiffer. H. Grunsky, 95 .

Curry, H. B. A theory of formal deducibility. D. Nelson, 415.

Curry, H. B. Leçons de logique algêbrique. R. Barcan Marcus, 673.

Dieudonne, J. See Nachbin, L.

Doetsch, G. Handbuch der Laplace- Transformation. Vol. 1. Theorie der LaplaceTransformation. E. Hille, 670.

Doetsch, G. See Voelker, D.

DuVal, P. See Segre, B.

Dwyer, P. S. Linear computations. H. H. Goldstine, 680.

Emory, F. L. See Laplace, P. S.

Erdélyi, A. See Schwank, F., Voelker, D.

Finsler, P. Über Kurven und Flächen in allgemeinen Räumen. With a bibliography by H. Schubert. H. Busemann, 102.

Garnier, R. Cours de cinématique. Vol. 1. Cinématique du point et du solide. Composition des mouvements. Vol. 2. Roulement et viration. La formule de Savary et son extension à l'espace. Vol. 3. Géométrie et cinématique cayleyennes. W. Blaschke, 504.

Geiringer, H. See Hill, R.

Gill, S. See Wilkes, M. V.

Goldstein, H. Classical mechanics. E. J. Moulton, 396.

Goldstine, H. H. See Dwyer, P. S.

Graves, L. M. See Lévy, P.

Grunsky, H. See Courant, R.

Hall, N. A. See König, R.

Halmos, P. R. Introduction to Hilbert space and the theory of spectral multiplicity. E. R. Lorch, 412.

Halperin, I. Introduction to the theory of distribution. Based on lectures by L. Schwarz. S. Bochner, 679.

Hardy, G. H., and Rogosinski, W. W. Fourier series, 419.

Heins, A. E. See de Broglie, L., Sneddon, I.

Heins, M. See Bieberbach, L.

Herland, L. Dictionary of mathematical sciences. Vol. 1. German-English. R. P. Boas, 505.

Hill, R. The mathematical theory of plasticity. H. Geiringer, 507.

Hille, E. See Doetsch, G., Sansone, G., Severi, F.

Hodge, P. G. See Prager, W.

Hodge, W. V. D., and Pedoe, D. Methods of algebraic geometry. Vol. 2. H.S.M. Coxeter, 678.

Jacobson, N. Lectures in abstract algebra. Vol. 1. Basic concepts. W. H. Mills, 579.

Jeffery, R. L. The theory of functions of a real variable. A. Rosenthal, 517.

Jourdain, P. E. B. See Cantor, G.

Kaplan, W. See McLachlan, N. W.

von Kármán, T. See Advances in applied mechanics.

Kleene, S. C. See Péter, R.

König, R., and Weise, K. H. Mathematische Grundlagen der höheren Geodäsie und Kartographie. Vol. 1. N.A. Hall, 98.

Koopmans, T. C. See Activity analysis of production and allocation.

Kuratowski, C. Topologie I. Espaces metrisables, espaces complets. G. T. Whyburn, 265.

Kuratowski, C. Topologie II. Espaces compacts, espaces connexes, plan euclidien. G. T. Whyburn, 265. 
Laplace, P. S. A philosophical essay on probabilities. Trans. by F. W. Truscott and F. L. Emory. With an introductory note by E. T. Bell. R. P. Boas, 682.

Levinson, N. See Titchmarsh, E. C.

Lévy, P. Problèmes concrets d'analyse fonctionelle. L. M. Graves, 408.

Lorch, E. R. See Halmos, P. R.

McKinsey, J. C. C. See Schmidt, A.

McLachlan, N. W. Ordinary non-linear differential equations in engineering and physical sciences. W. Kaplan, 583.

Massey, W. S. See Colloque de Topologie.

Mills, W. H. See Jacobson, N.

von Mises, R. See Advances in applied mechanics.

Moulton, E. J. See Banach, S., Goldstein, H.

Murnaghan, F. D. Finite deformation of an elastic solid. C. Truesdell, 577.

Nachbin, L. Espaços vetoriais topologicos 1. J. Dieudonné, 268.

Nagell, T. Introduction to number theory. L. Schoenfeld, 580.

Nehari, Z. Conformal mapping. G. Springer, 515.

Nelson, D. See Curry, H. B.

Newman, M. H. A. Elements of the topology of plane sets of points. P. A. Smith, 101.

Novak, I. L. See Rosenbloom, P. C.

Ostrowski, A. Vorlesungen ilber Differential- und Integralrechnung. Vol. II. Differentialrechnung auf dem Gebiete mehrerer Variablen. A. E. Taylor, 513.

Pedoe, D. See Hodge, W. V. D.

Péter, R. Rekursive Funktionen. S. C. Kleene, 270.

Prager, W., and Hodge, P. C. Theory of perfectly plastic solids. C. Truesdell, 674.

Proceedings of a Second Symposium on Large-Scale Digital Calculating Machinery. J. Todd, 418.

Proceedings of the International Congress of Mathematicians, 419.

Proceedings of the Second Canadian Mathematical Congress, Vancouver, 1949, 280.

Rogosinski, W. W. See Hardy, G. H.

Rosenbloom, P. C. The elements of mathematical logic. I. L. Novak, 266.

Rosenthal, A. See Jeffery, R. L.

Ruthishauser, H., Speiser, A., and Steifel, E. Programmgesteuerte digitale Rechengeräte (Elektronische Rechenmaschinen). J. Todd, 278.

Salzer, H. E. Tables of $n !$ and $\Gamma(n+1 / 2)$ for the first thousand values of $n, 279$.

Sansone, G. Moderna teoria delle funzioni di variabile reale. Part II. Sviluppi in serie di funzioni ortogonali. E. Hille, 681.

Sansone, G. See Vitali, G.

Schiffer, M. See Courant, R.

Schmidt, A. Mathematische Grundlagenforschung. J. C. C. McKinsey, 94.

Schoenfeld, L. See Nagell, T.

Schubert, H. See Finsler, P.

Schwank, F. Randwertprobleme und andere Anwendungsgebiete der Höheren Analysis für Physiker, Mathematiker und Ingenieure. A. Erdélyi, 274.

Schwartz, L. Théorie des distributions. S. Bochner, 78.

Schwartz, L. See Halperin, I.

Scorza Dragoni, G. See Severi, F.

Scott, E. J. See Banach, S.

Segre, B. Arithmetical questions on algebraic varieties. P. DuVal, 575.

Severi, F., and Scorza Dragoni, G. Lezioni di analisi. Vol. 3. E. Hille, 417.

Smith, P. A. See Newman, M. H. A.

Sneddon, I. Fourier transforms. A. E. Heins, 512.

Speiser, A. See Ruthishauser, H.

Springer, G. See Nehari, Z.

Stiefel, E. See Ruthishauser, H. 
Stoker, J. J. See Birkhoff, G.

Tables of the error function and of its first twenty derivatives, 279.

Tables relating to Mathieu functions. Characteristic values, coefficients, and joining factors. C. J. Bouwkamp, 85.

Taylor, A. E. See Ostrowski, A.

Titchmarsh, E. C. The theory of the Riemann zeta-function. N. Levinson, 401.

Todd, J. Tables of arctangents of rational numbers, 279.

Todd, J. See Proceedings of a Second Symposium on Large-Scale Digital Calculating Machinery, Ruthishauser, H., Wilkes, M. V.

Tranter, C. J. Integral transforms in mathematical physics. R. V. Churchill, 102.

Tricomi, F. Funzioni ellittiche.

Truesdell, C. See Advances in applied mechanics, Murnaghan, F. D., Prager, W.

Truscott, F. W. See Laplace, P. S.

Vitali, G. and Sansone, G. Moderna teoria delle funzioni di variable reale, 103.

Voelker, D., and Doetsch, G. Die zweidimensionale Laplace-Transformation. A. Erdélyi, 88.

Weise, K. H. See König, R.

Wheeler, D. J. See Wilkes, M. V.

Whyburn, G. T. See Kuratowski, C.

Wilkes, M. V., Wheeler, D. J., and Gill, S. The preparation of programs for an electronic digital computer. J. Todd, 276.

Wolfowitz, J. See Activity analysis of production and allocation.

\section{INVITED ADDRESSES ${ }^{1}$}

Bing, R. H. Partitioning continuous curves, 536.

Bourgin, D. G. Errata to Classes of transformations and bordering transformations, 696.

Chowla, S. The Riemann zeta and allied functions, 287.

Federer, H. Measure and area, 306.

Forsythe, G. E., 559.

Gödel, K., 158.

Hochschild, G. P., 159.

Jones, F. B. Concerning aposyndetic and non-aposyndetic continua, 137.

Kakutani, S., 159.

Kelley, J. L., 50.

Kolchin, E. R., 440.

McKinsey, J. C. C., 62.

Some notations and problems of game theory, 591.

Moise, E. E., 613.

Morse, M., 439.

Oxtoby, J. C., 38. Ergodic sets, 116.

Robbins, H., 50.

Some aspects of the sequential design of experiments, 527.

Rothe, E. H., 152.

Samelson, H. Topology of Lie groups, 2.

Schoenberg, I. J., 493.

Tarski, A., 613.

Ulam, S. M., 472.

Weinstein, A., 379.

${ }^{1}$ Where the title of the address is not given, the reference is to the Report of the Meeting at which the address was given. 